\title{
Endothelial fluid shear stress sensing in vascular health and disease
}

\author{
Nicolas Baeyens, ${ }^{1}$ Chirosree Bandyopadhyay, ${ }^{1}$ Brian C. Coon, ${ }^{1}$ Sanguk Yun, ${ }^{1}$ and Martin A. Schwartz ${ }^{1,2}$ \\ 'Yale Cardiovascular Research Center, Department of Medicine (Cardiology), and 'Departments of Cell Biology and Biomedical Engineering, Yale University, New Haven, Connecticut, USA.
}

Endothelial cells transduce the frictional force from blood flow (fluid shear stress) into biochemical signals that regulate gene expression and cell behavior via specialized mechanisms and pathways. These pathways shape the vascular system during development and during postnatal and adult life to optimize flow to tissues. The same pathways also contribute to atherosclerosis and vascular malformations. This Review covers recent advances in basic mechanisms of flow signaling and the involvement of these mechanisms in vascular physiology, remodeling, and these diseases. We propose that flow sensing pathways that govern normal morphogenesis can contribute to disease under pathological conditions or can be altered to induce disease. Viewing atherosclerosis and vascular malformations as instances of pathological morphogenesis provides a unifying perspective that may aid in developing new therapies.

\section{Introduction}

Mechanical forces guide the development and physiology of every organ system, but they are particularly crucial in the cardiovascular system, whose main function of transporting pressurized blood to every tissue in the body is essentially mechanical. Blood pumped from the heart exerts two main forces on the vasculature: the frictional force from blood flow (fluid shear stress [FSS]) that is parallel to the vessel wall, and the force from blood pressure that stretches the vessel wall. These forces are sensed and interpreted by the cells of the vessel wall to guide development during embryogenesis and remodeling during postnatal and adult life to optimize blood flow to the tissues and to ensure the mechanical integrity of the vessel walls. However, when either the forces themselves or the mechanisms by which forces are sensed are pathological, mechanotransduction contributes to the major diseases of the vasculature.

This review briefly discusses basic pathways of FSS mechanotransduction, then how these pathways govern physiological remodeling, and last, how these same mechanisms contribute to disease. Our overarching view is that pathways evolved for developmental and physiological regulation are subverted or altered in disease. The emphasis of this Review is on how recent advances in the understanding of mechanotransduction will elucidate pathological processes and might offer new paths to therapies.

\section{Basic mechanisms of FSS signaling}

The ability of the vascular system to perfuse all tissues in the body requires precise control of blood flow through each vascular bed. Endothelial sensing of FSS governs both short-term vascular tone and long-term vascular remodeling to adjust vessel diameters to tissue demand. Endothelial cells (ECs) have

Authorship note: N. Baeyens, C. Bandyopadhyay, B.C. Coon, and S. Yun contributed equally to this work and are listed in alphabetical order

Conflict of interest: The authors have declared that no conflict of interest exists.

Reference information: J Clin Invest. 2016;126(3):821-828. doi:10.1172/JCI83083. evolved sophisticated mechanosensing abilities to detect distinct features of flow profiles and regulate vessel physiology and remodeling accordingly (1). These features include flow magnitude, direction, and the amplitude and frequency of pulsatile flow (2-6). In humans, normal physiological flow is between roughly 1 and 5 pascals $(\mathrm{Pa})$ and is highly pulsatile in arteries; of similar magnitude but less pulsatile in capillaries; and about 10-fold less with minimal pulsatility in veins (7). These forces are very low compared to typical traction stresses $(\sim 5 \mathrm{kPa}$; ref. 8$)$ and artery wall strains (on the order of $100 \mathrm{kPa}$; ref. 9), underscoring the sensitive nature of these endothelial-specific mechanisms. Flow induces dose-dependent secretion of nitric oxide (NO) and prostacyclin, which relax smooth muscle and decrease vascular tone, a homeostatic mechanism that maintains a constant FSS (10, 11). Flow also induces dose-dependent expression of the transcription factor Krüppel-like factor 2 (KLF2), which induces the expression of multiple antiinflammatory, antithrombotic, and antioxidative mediators to stabilize the vessel wall in adults (12). While FSS within the physiological range stabilizes blood vessels $(12,13)$, prolonged low or high flow leads to inward or outward remodeling of the vessel wall, respectively (14-17). Regions of arteries with bifurcations, curvatures, or valves have weak flow with complex changes in direction (collectively termed disturbed flow), which, as discussed below, makes them susceptible to the formation of atherosclerotic lesions. Occlusions from atherosclerosis also give rise to very high flow at the narrow region and low flow downstream, which affect lesion progression.

Elucidation of FSS pathways is ongoing. In vitro studies have catalogued a wide range of shear-responsive kinases, GTPases, ion channels, and other signaling molecules and signaling events, as well as many downstream genes and microRNAs (reviewed in ref. 13). Recently, epigenetic marks have also been observed $(18,19)$. Of particular relevance for understanding disease, most studies have primarily focused on differential effects of onset of physiological levels of steady laminar flow as a model for stable vessels versus oscillatory shear stress as a model for 
atherosclerosis. Physiological laminar shear promotes cell elongation and orientation in the direction of flow, suppresses proliferation, stimulates antiinflammatory gene expression, and suppresses expression of inflammatory pathways (20-22). Some evidence indicates that cells are sensitive to features of pulsatile flow $(23,24)$, though more systematic studies of these effects are needed. By contrast, oscillatory shear stress activates inflammatory pathways and suppresses expression of the antiinflammatory, atheroprotective transcription factor KLF2 (reviewed in refs. 12, 25).

Upstream of these events, many mechanotransducers have been proposed, including PECAM-1, the apical glycocalyx, ion channels, G proteins, protein kinases, and primary cilia (26-29). However, a coherent model of flow sensing is lacking. The beststudied mechanotransducer is a complex of endothelial-specific proteins localized at cell-cell junctions, consisting of PECAM-1, VE-cadherin, and two VEGF receptors, VEGFR2 and VEGFR3 (30-35). Recent work showed that flow increases tension on PECAM-1 via an upstream pathway that triggers association of PECAM-1 with the vimentin cytoskeleton, thereby transmitting force from myosin to PECAM-1 (32). Tension on PECAM-1 triggers activation of a Src family kinase, resulting in ligand-independent transactivation of VEGF receptors and subsequent activation of PI3K, endothelial NOS (eNOS), and production of NO to induce vasodilation $(31,36)$. PI3K also activates integrins, which mediate another important subset of flow-dependent pathways involved in cell alignment in laminar flow and activation of inflammatory pathways in disturbed flow (31). VE-cadherin's role in this pathway appeared to be as an adapter rather than force transducer $(31,32)$. Mapping studies showed that VE-cadherin associated with VEGF receptors through their respective transmembrane domains, which was required for VEGFR transactivation and downstream signaling (33). The mechanosensor upstream of PECAM-1 has not been identified; however, $G \alpha_{q / 11}$ proteins were reported to co-immunoprecipitate with PECAM-1, and this interaction was diminished after onset of flow (37). Recent data showed that flow triggers secretion of ATP from the cells, inducing autocrine or paracrine activation of its $\mathrm{G} \alpha_{q / 11}$-coupled purine receptor, $\mathrm{P} 2 \mathrm{Y}_{2}$ (27). Deletion or inhibition of either $\mathrm{P}_{2} \mathrm{Y}_{2}$ or $\mathrm{G \alpha}_{\mathrm{q} / 11}$ inhibited flow-induced calcium transients and phosphorylation of PECAM-1, VEGFR2, and eNOS in vitro. Additionally, acute deletion of these genes elevated blood pressure in vivo, consistent with a reduction in NO production. Thus, $\mathrm{P} 2 \mathrm{Y}_{2}$ and $\mathrm{G} \alpha_{\mathrm{q} / 11}$ are excellent candidates for upstream mediators of PECAM-1 cytoskeletal association and mechanotransduction.

\section{Physiological remodeling}

It has long been proposed that vessels undergo long-term remodeling that maintains FSS within a narrow range, often termed a set point (38). For example, surgical manipulations, changes in tissue demand, or arterial blockages that increase or decrease flow through a vessel result in outward or inward remodeling that return FSS closer to the original set point values (39). These longterm changes are partly due to a process called entrenchment, where vasoconstriction or dilation are entrenched through matrix remodeling. However, tissue remodeling also involves inflamma- tion in which newly recruited monocytes and macrophages contribute cytokines, metalloproteinases, and extracellular matrix (ECM) proteins that are essential for the process $(40,41)$. A recent study showed that ECs exhibit an FSS set point such that flow within the physiological range suppresses inflammatory signaling and activates pathways that stabilize the vessel, whereas flow below or above the physiological range activates inflammatory pathways and suppresses the stabilization pathways (2). The set point model implies that different types of vessels (arteries, veins, lymphatics) that have very different flow magnitudes and patterns must have different set points. This study also showed that differences in expression of VEGFR3, a component of the junctional complex that includes PECAM-1 and VE-cadherin (33), could explain most of the difference in set point between blood ECs and lymphatic ECs, where flow is much lower. Deletion of PECAM-1 inhibits both low flow/inward remodeling and high flow/outward remodeling $(42,43)$, supporting a role for the junctional complex in these processes.

While low shear/inward remodeling and high shear/outward remodeling both involve activation of inflammatory pathways $(2,44)$, what determines the direction of remodeling is poorly understood, despite its importance for patients with arterial restrictions. High flow has been shown to induce a distinct gene expression profile relative to normal flow; this expression profile contains both antiinflammatory and remodeling genes (45). Outward remodeling in high flow has also been shown to require both the NADPH oxidase complex and eNOS (46). The identity of pathways that distinguish these two processes to determine the direction of remodeling remains a key, clinically relevant question.

\section{Atherosclerosis}

Atherosclerosis is a chronic inflammatory disease of the arteries in which plaques develop in regions where flow is disturbed due to vessel geometry $(12,47,48)$. Straight segments of arteries with unidirectional, high laminar shear are actively protected from atherosclerosis due to induction of antiinflammatory, antioxidative, and antithrombotic genes, in large part through induction of the flow-dependent transcription factors KLF2 and nuclear factor (erythroid-derived 2)-like 2 (NRF2) (12). By contrast, regions of arteries where time-averaged shear stress is low and subject to complex directional changes exhibit low, chronic inflammation even under control conditions, such as in WT mice that do not develop atherosclerosis (49). This "primed" state associated with low, disturbed flow is not strongly inflamed but makes the endothelium more susceptible to risk factors such as high LDL cholesterol and hyperglycemia. These effects have been corroborated in many in vitro studies showing that low or oscillatory shear stress fails to induce EC alignment in the direction of flow and moderately activates multiple inflammatory events including increased permeability, ROS generation, NF- $\kappa$ B activity, and expression of receptors and cytokines that recruit leukocytes. Perhaps more importantly, this preexisting state sensitizes ECs to the action of other inflammatory mediators (12). These results are consistent with studies showing that disease risk in vivo correlates with regions of low flow magnitude and with various metrics of flow disturbance (50-52). 
Susceptibility to atherosclerosis also correlates with failure of the ECs to align in the flow direction (53). This finding has generally been attributed to low and/or oscillatory shear (49). Recently, however, it has been suggested that transverse flow (i.e., flow at $90^{\circ}$ to the vessel axis) shows the best correlation with plaque formation over a range of conditions in animal models (52). This in vivo result fits well with an in vitro study showing that flow perpendicular to the morphological and cytoskeletal axis of ECs activated inflammatory pathways, whereas flow that was parallel was antiinflammatory, even if it reversed direction (54). The inflammatory effect of low or oscillatory flow can be rationalized by the fact that cells fail to align in these conditions; thus, even unidirectional flow will have a transverse component for a fraction of the cell population. This idea also fits well with the observation that onset of unidirectional laminar shear initially activates the inflammatory pathways, but these pathways are downregulated at later times as cells align and the antiinflammatory pathways become dominant (55). The mechanistic basis for these effects is unknown, but a tantalizing clue comes from the finding that production of ROS by the NADPH oxidase complex in response to flow requires association of VE-cadherin with the oxidase complex through the polarity protein PAR3 (56). A link between alignment and atherosclerosis in vivo is supported by studies on syndecan $4^{-/-}$mice, in which ECs fail to align in flow; on a hypercholesterolemic background, these mice show increased atherosclerosis, including lesions in regions of laminar flow that are normally protected (57).

Disturbed flow patterns induce inflammatory activation of the endothelium through a remarkably broad array of pathways and genes; see refs. 12, 49, for excellent reviews on this subject. More recent work implicates multiple microRNAs in this process (58, 59). A fascinating connection between flow patterns, microRNAs, and lipid metabolism comes from studies of the cholesterolregulated transcription factor sterol regulatory element-binding protein 2 (SREBP2). Disturbed flow activates SREBP2 (without changes in cellular cholesterol content), which mediates activation of the NRLP3 inflammasome (60). Activation of this pathway results in IL-1 $\beta$ production, an important contributor to atherosclerosis that is currently targeted in clinical trials (61). Effects of SREBP2 require expression of miR-92a, which targets a number of atheroprotective mediators (62). Despite the diversity of inflammatory events triggered by disturbed flow, it should be kept in mind that the effects are not one-sided. Disturbed flow also activates important negative regulators that suppress inflammation (53). These findings are hardly surprising in light of the benign nature of disturbed flow in the absence of other risk factors. Even with the presence of risk factors, disease generally takes many decades to manifest clinically.

An interesting connection between atherosclerosis and physiological flow-dependent remodeling can be observed at the level of ECM remodeling. Normal endothelial basement membranes are composed mainly of collagen IV, laminin, and other glycoproteins and proteoglycans. Both flow-dependent remodeling and sprouting angiogenesis require proteolysis of this ECM and assembly of a fibronectin matrix (63-65). Fibronectin also accumulates beneath the endothelium in atheroprone regions of arteries in normal adult mice, colocalized with inflammatory markers such as ICAM-1 or VCAM-1 (66). Mechanistic in vitro studies have shown that fibronectin controls activation of multiple inflammatory mediators in response to disturbed flow (66, 67) as well as oxidized LDL, another important inflammatory mediator in atherosclerosis (68). Furthermore, fibronectin suppresses activation of eNOS and production of $\mathrm{NO}$ in response to flow (69), thereby inducing endothelial dysfunction. The importance of fibronectin in atherosclerosis is supported by animal studies demonstrating that genetic manipulations to reduce fibronectin in the vessel wall reduce plaque burden (70-72). Interestingly, diabetes, which is a potent risk factor for atherosclerosis, further increases fibronectin deposition in atherosclerosis-prone regions of arteries (73).

Atherosclerosis may thus be seen as a form of flow-dependent remodeling that never resolves. This idea could be tested by examining genetically modified mice with specific defects in flow sensing in both flow-dependent remodeling and atherosclerosis. As noted above, work on Pecam1 $1^{-/-}$mice supports this connection; however, PECAM-1 is expressed in hematopoietic cells and has multiple functions in all cells, which complicates the interpretation of these data. Identification of genes in humans and mice that control growth of collateral circulation after arterial blockage will allow comparison to the genes that control atherosclerosis and will provide a further, unbiased test of this hypothesis.

\section{Plaque progression and remodeling}

Once plaques are formed, mechanical factors contribute to their continued remodeling. Intrusion of the plaque into the vessel lumen creates a region of low/disturbed flow downstream, which may account for the tendency of plaques to progress in a downstream direction $(74,75)$. Installation of a cuff on carotid arteries in hypercholesterolemic mice creates an upstream region of low, unidirectional flow and a downstream region of oscillatory flow (76). Plaques formed in the upstream segments showed increased levels of markers of vulnerability, whereas the downstream region appeared more stable; however, the pressure drop across the restriction complicates the interpretation of these results. Thus, it is unknown to what extent high blood pressure contributes to the vulnerable phenotype in the upstream low-flow region compared to the downstream, oscillatory, low-pressure regions. In other models, both low shear and transverse shear were strongly correlated with regions of vulnerability within plaques (77-79). Plaque rupture itself also depends on wall strain. In particular, regions of low shear stress but high strain appear to be at greatest risk, presumably because of the combination of vulnerable plaque and high strain (80). Microcalcification within a thin fibrous cap can also increase the risk of rupture, probably because of compliance mismatch, which generates regions of especially high strain (81).

Atherosclerotic lesions can restrict the artery lumen, but vessels respond by outward remodeling to maintain lumen diameter $(82,83)$. This process, often called the Glagov phenomenon, is a specific example of the FSS set point theory: blood vessels remodel to maintain a constant FSS $(2,38)$. However, this beneficial remodeling fails as lesions enlarge, usually at around $40 \%$ stenosis, after which plaque enlargement obstructs the vessel. One explanation for the failure to maintain lumen diameter is that fibrous connec- 
tive tissue of the adventitia could serve as a physical barrier to further expansion and stiffening of the adventitia under loading (84) or local inflammation, and thickening of the adventitia in atherosclerosis (85) could contribute to this effect. An alternative explanation is that the endothelium undergoes changes that inhibit flow sensing and/or remodeling. The recent observation that ECs undergo an endothelial-mesenchymal transition (EndMT) in response to proatherogenic flow and inflammatory cytokines present in atherosclerotic lesions might also explain this phenomenon $(86,87)$. EndMT results in reduced expression of endothelial-specific molecules including VE-cadherin and PECAM-1, which mediate flow sensing and could prevent flow-dependent remodeling. This hypothesis is corroborated by the observation that vein graft stenosis following exposure to the arterial environment, with consequent failed venous graft adaptation and remodeling, is characterized by SMAD2/3-dependent EndMT (88).

Outward remodeling has been linked to plaque vulnerability $(89,90)$, perhaps through increased expression of metalloproteinases that mediate remodeling but also degrade collagen in the fibrous cap. However, a recent large-cohort study addressing the role of lesion remodeling in major adverse cardiac events found that lesions with multiple necrotic cores and thin fibrous caps were more prevalent in the context of inward remodeling (91). This study also highlighted the common occurrence (approximately $95 \%$ of patients) of both negative and positive remodeling of lesions within the same patient, emphasizing the role of local factors in determining lesion remodeling.

\section{Collateral growth and flow-dependent remodeling}

Upon artery occlusion, blood flow is redirected into smaller collateral vessels upstream of the lesion site, which induces enlargement of these small vessels, to improve perfusion of the surrounding tissue (92). Increased collateral flow is also promoted by the arterialization of preexisting capillaries $(93,94)$. Collateral circulation, characterized clinically as the collateral flow index, is a major determinant of favorable outcome after myocardial infarction. This form of flow-dependent remodeling is mediated by activation of NF-кB (2), induction of ICAM-1 (95), and local recruitment of monocytes/macrophages that secrete VEGF and other factors that promote arterialization of capillaries $(96,97)$. Interestingly, two noninvasive therapeutic approaches to increase FSS in the coronary vascular network are being tested to promote collateral flow: physical reeducation (under trial, ref. 98) and external counterpulsation with pneumatic cuffs $(99,100)$.

Why some patients do not develop proper collateral vascularization after myocardial infarction remains a major clinical question. In mice, different strains exhibit striking variation in the extent of remodeling and recovery following femoral artery ligation (101). Genetic mapping comparing the two strains at the extremes of the distribution for collateral arteriogenesis restrained the possible differences to a few genetic loci, though no candidate gene that explains the differences has been identified (102). Hypercholesterolemia $(103,104)$, obesity (105), and aging (106) also compromise arteriogenesis in mouse models. Thus, poor flow-dependent remodeling in human arterial disease is likely to have multiple causes, potentially requiring personalized treatment.

\section{Vascular malformations}

Vascular malformations are structurally anomalous vessels that cause leakage, hemorrhage, and loss of capillary function (107109). One clinically important form is cerebral cavernous malformations (CCMs), which are low-flow vascular malformations (as opposed to arterial-venous malformations, which usually have high flow) that affect up to 1 in 200 people, causing neurological deficits in younger individuals and stroke in midlife. CCM is frequently an autosomal dominant inherited disease due to heterozygous mutations in one of three genes: CCM1 (KRIT1), CCM2, and CCM3 (PDCD10) $(108,110)$. Patients acquire sporadic lesions with age, presumably due to additional mutations or other local stresses, though these additional events are not well characterized. The CCM proteins are expressed in ECs, where they localize mainly at cell-cell junctions, anchored to the membrane in part through the transmembrane receptor heart-of-glass. Mouse genetic studies have shown that all three CCM proteins are required for embryonic and postnatal cardiovascular development (111). Clinical features of the disease and the biochemistry of the proteins and their interactions have been reviewed previously (112).

Dilated vessels with low flow will have even lower shear stress due to the inverse dependence of FSS on vessel diameter, raising the question of how the normal homeostatic control of vessel diameter is circumvented in CCM. Indeed, recent studies have connected CCM proteins to flow signaling. First, deletion of CCM1 in ECs results in dramatic upregulation of the transcription factors KLF2 and -4 (113), which are normally induced by flow (12). CCM mutation or deletion results in similar upregulation of KLF2 in mice, zebrafish, and cultured human ECs, and similar developmental heart defects in mice and zebrafish. In zebrafish, reduction of Klf2 partially rescued developmental defects. Klf2 upregulation was due to increased Mekk3 kinase activity, which is upstream of Erk5, the kinase that mediates flow induction of Klf2. Intriguingly, Mekk3 associates with the CCM complex, and its deletion also rescued the developmental defects. Together, these data suggest the hypothesis that FSS might work by relieving the inhibitory effect of the CCM complex on Mekk3 to activate Erk5 and downstream events (113). This idea is at present speculative but attractive in light of the role of cell-cell junctions in shear mechanotransduction. It should be appreciated that these conclusions are based partly on studies (114) of heart development induced by complete deletion at early stages, which is distinct in many respects from the adult-onset vascular malformations associated with heterozygous mutations. However, these systems may differ primarily in the events downstream of Erk5 and Klf2, which promote angiogenesis in development but promote vessel stability in adults (114). It is also likely that other unique features of brain vasculature are critical; secretion of BMP6 in CCMs appears to be one such event (115). While the biology of lesions clearly is complex, understanding the molecular connections between flow, CCM proteins, and the Klf2 pathway will be an interesting area for investigation.

A second potential connection involves the direct binding of CCM1 to integrin cytoplasmic domain-associated protein-1 (ICAP1), a protein that inhibits the conformational activation of $\beta_{1}$ integrins and increases their binding affinity for ligands (116). This interaction stabilizes ICAP1 so that loss of the CCM complex results in decreased ICAP1 levels and increased integrin activity. Flow also activates integrins, which mediate an important subset 


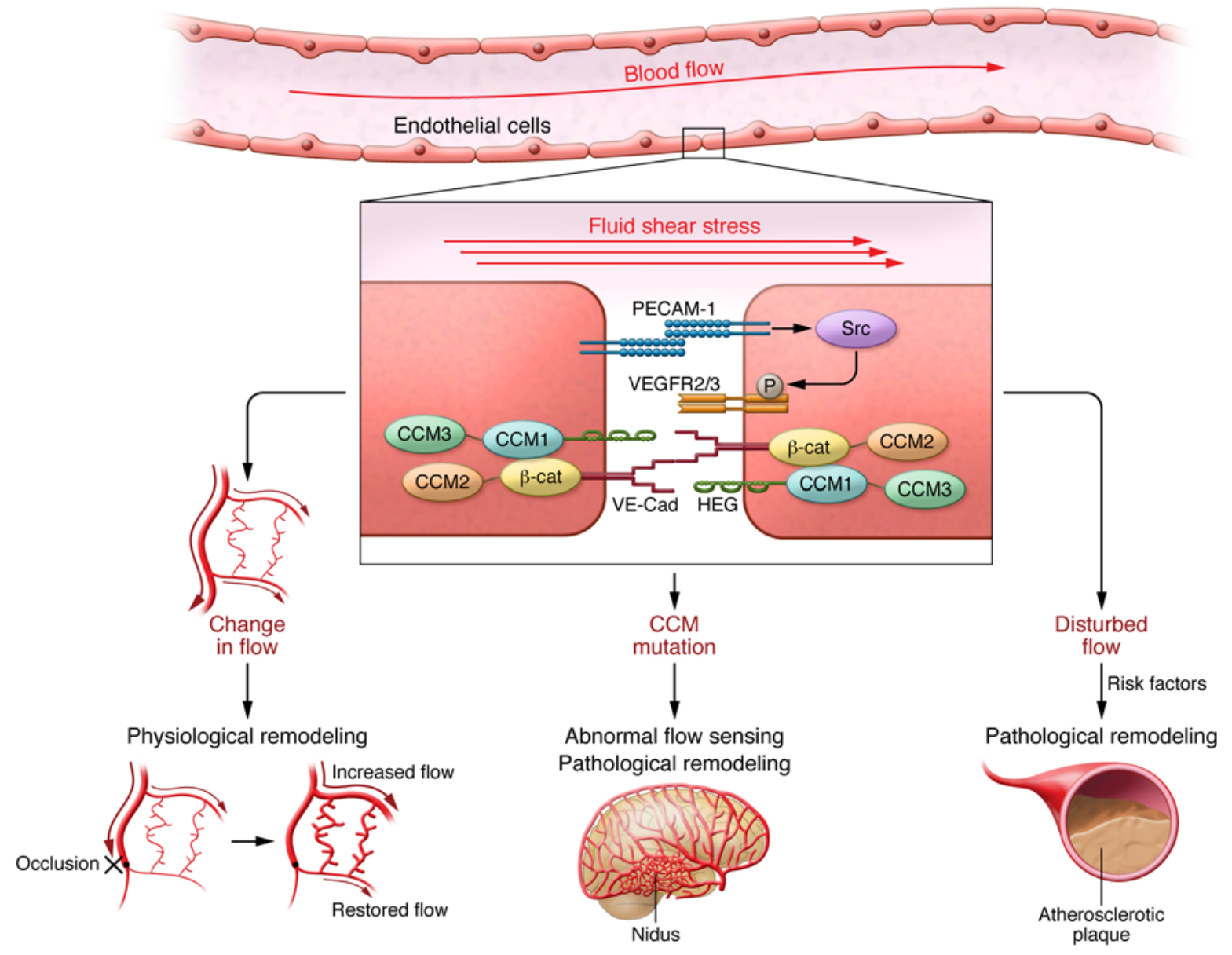

Figure 1. Fluid shear stress sensing in physiological and pathological vascular remodeling. FSS acts on mechanosensors including the junctional mechanosensory complex consisting of PECAM-1, VEGFRs, and VE-cadherin (VE-Cad), with the latter also linked to the CCM complex (CCM1, CCM2, and CCM3). Left: Changes in flow due to growth or exercise that result in sustained deviation from the set point result in changes in the activation state of flowdependent pathways, which mediate physiological remodeling to restore normal shear stress levels. Center: Mutations in CCM genes results in abnormal flow sensing and pathological remodeling, with subsequent formation of abnormal, low-flow vessels. Right: Regions of disturbed flow, in the presence of systemic risk factors, result in pathological remodeling to form atherosclerotic plaque. $\beta$-cat, $\beta$-catenin; HEG, heart-of-glass.

of flow-dependent pathways (117). Indeed, more recent data show that loss of the CCM complex induces flow-independent integrin activation (118) and that integrins are required for KLF2 induction (119). These data are again consistent with the general idea that loss of CCM proteins results in constitutive activation of a pathway that is normally regulated by flow. In this regard, it is also interesting that CCMs have now been linked to EndMT (115). This transition required upregulation of BMP6, which occurred specifically in brain vasculature, perhaps explaining the specificity of CCMs for this location. On one hand, disturbed flow can induce EndMT $(86,87)$. Conversely, downregulation of EC-specific proteins such as VE-cadherin and PECAM-1, which participate in flow signaling, would be expected to reduce flow responsiveness. There are a number of different ways in which these observations might be related. Either low flow or low sensitivity to flow in malformations might sensitize the endothelium to the action of BMP6 or otherwise contribute to EndMT. EndMT might also contribute to loss of flow sensitivity. In either case, the critical role of flow in regulation of vascular morphogenesis, including both vessel diameter and stabilization, suggests multiple mechanisms by which flow independence could contribute to vascular malformations. Further investigation into blood flow patterns in lesions and the effect of CCM mutations and pathways on flow sensing are warranted.

\section{Conclusions}

Vascular development and remodeling are morphogenetic processes that depend on many genes but are guided by mechanical forces from blood flow. This feature very likely reflects the need to optimize vessel morphology to carry blood with maximum efficiency. Sensing of FSS by ECs is a key element of these processes. Our understanding of FSS signaling, though largely incomplete, has reached a stage in which connections to disease are coming into focus. We proposed that atherosclerosis and vascular malformations are, in essence, diseases of morphogenesis. That is, normal morphogenetic programs have gone awry, resulting in the generation of vessels with abnormal characteristics (summarized in Figure 1). Atherosclerosis may be seen as a form of futile vascular remodeling that occurs in an inflammatory environment at regions of disturbed shear. CCMs may be seen as the result of genetic mutations that disrupt normal homeostasis, of which flow sensing is a key component. Future research is likely to benefit from walking this two-way street, in which genes and processes involved in 
disease may shed light on basic cellular mechanisms, and understanding basic mechanisms may lead the way to understanding pathogenesis and ultimately developing new treatments.

\section{Acknowledgments}

This work was supported by US Public Health Service grants R01 HL75092 and P01 HL107205 to M.A. Schwartz and by Amer- ican Heart Association postdoctoral fellowships to N. Baeyens (14POST19020010) and B.G. Coon (13POST16720007).

Address correspondence to: Martin A. Schwartz, Cardiovascular Research Center, 300 George St., $7^{\text {th }}$ floor, New Haven, Connecticut 06511, USA. Phone: 203.737.2930; E-mail: martin.schwartz@ yale.edu.
1. Davies PF. Flow-mediated endothelial mechanotransduction. Physiol Rev. 1995;75(3):519-560.

2. Baeyens $\mathrm{N}$, et al. Vascular remodeling is governed by a VEGFR3-dependent fluid shear stress set point. Elife. 2015;4.

3. Noris M, et al. Nitric oxide synthesis by cultured endothelial cells is modulated by flow conditions. Circ Res. 1995;76(4):536-543.

4. Wang C, Baker BM, Chen CS, Schwartz MA. Endothelial cell sensing of flow direction. Arterioscler Thromb Vasc Biol. 2013;33(9):2130-2136.

5. Blackman BR, Garcia-Cardena G, Gimbrone MA Jr. A new in vitro model to evaluate differential responses of endothelial cells to simulated arterial shear stress waveforms. J Biomech Eng. 2002;124(4):397-407.

6. Feaver RE, Gelfand BD, Blackman BR. Human haemodynamic frequency harmonics regulate the inflammatory phenotype of vascular endothelial cells. Nat Commun. 2013;4:1525.

7. Paszkowiak JJ, Dardik A. Arterial wall shear stress: observations from the bench to the bedside. Vasc Endovascular Surg. 2003;37(1):47-57.

8. Balaban NQ, et al. Force and focal adhesion assembly: a close relationship studied using elastic micropatterned substrates. Nat Cell Biol. 2001;3(5):466-472.

9. Humphrey JD, Dufresne ER, Schwartz MA. Mechanotransduction and extracellular matrix homeostasis. Nat Rev Mol Cell Biol. 2014;15(12):802-812.

10. Joannides $R$, et al. Nitric oxide is responsible for flow-dependent dilatation of human peripheral conduit arteries in vivo. Circulation. 1995;91(5):1314-1319.

11. Frangos JA, Eskin SG, McIntire LV, Ives CL. Flow effects on prostacyclin production by cultured human endothelial cells. Science. 1985;227(4693):1477-1479.

12. Nigro P, Abe J, Berk BC. Flow shear stress and atherosclerosis: a matter of site specificity. Antioxid Redox Signal. 2011;15(5):1405-1414.

13. Zhou J, Li YS, Chien S. Shear stress-initiated signaling and its regulation of endothelial function. Arterioscler Thromb Vasc Biol. 2014;34(10):2191-2198.

14. Kamiya A, Togawa T. Adaptive regulation of wall shear stress to flow change in the canine carotid artery. Am J Physiol. 1980;239(1):H14-H21.

15. Tronc F, Wassef M, Esposito B, Henrion D, Glagov $\mathrm{S}$, Tedgui A. Role of NO in flow-induced remodeling of the rabbit common carotid artery. Arterioscler Thromb Vasc Biol. 1996;16(10):1256-1262.

16. Tuttle JL, et al. Shear level influences resistance artery remodeling: wall dimensions, cell density, and eNOS expression. Am J Physiol Heart Circ Physiol. 2001;281(3):H1380-H1389.

17. Langille BL, O'Donnell F. Reductions in arterial diameter produced by chronic decreases in blood flow are endothelium-dependent. Science. 1986;231(4736):405-407.

18. Dunn J, Simmons R, Thabet S, Jo H. The role of epigenetics in the endothelial cell shear stress response and atherosclerosis. Int J Biochem Cell Biol. 2015;67:167-176.

19. Jiang YZ, Manduchi E, Jimenez JM, Davies PF. Endothelial epigenetics in biomechanical stress: disturbed flow-mediated epigenomic plasticity in vivo and in vitro. Arterioscler Thromb Vasc Biol. 2015;35(6):1317-1326.

20. Garcia-Cardena G, Comander J, Anderson KR, Blackman BR, Gimbrone MA Jr. Biomechanical activation of vascular endothelium as a determinant of its functional phenotype. Proc Natl Acad Sci U S A. 2001;98(8):4478-4485.

21. SenBanerjee $S$, et al. KLF2 Is a novel transcriptional regulator of endothelial proinflammatory activation. JExp Med. 2004;199(10):1305-1315.

22. Chappell DC, Varner SE, Nerem RM, Medford RM, Alexander RW. Oscillatory shear stress stimulates adhesion molecule expression in cultured human endothelium. Circ Res. 1998;82(5):532-539.

23. Kadohama T, Nishimura K, Hoshino Y, Sasajima $\mathrm{T}$, Sumpio BE. Effects of different types of fluid shear stress on endothelial cell proliferation and survival. J Cell Physiol. 2007;212(1):244-251.

24. Zhang Y, et al. AMP-activated protein kinase is involved in endothelial NO synthase activation in response to shear stress. Arterioscler Thromb Vasc Biol. 2006;26(6):1281-1287.

25. Davies PF. Hemodynamic shear stress and the endothelium in cardiovascular pathophysiology. Nat Clin Pract Cardiovasc Med. 2009;6(1):16-26.

26. Li YS, Haga JH, Chien S. Molecular basis of the effects of shear stress on vascular endothelial cells. J Biomech. 2005;38(10):1949-1971.

27. Wang S, et al. P2Y2 and Gq/G11 control blood pressure by mediating endothelial mechanotransduction. J Clin Invest. 2015;125(8):3077-3086.

28. Goetz JG, et al. Endothelial cilia mediate low flow sensing during zebrafish vascular development. Cell Rep. 2014;6(5):799-808.

29. Hahn C, Schwartz MA. Mechanotransduction in vascular physiology atherogenesis. Nat Rev Mol Cell Biol. 2009;10(1):53-62.

30. Osawa M, Masuda M, Harada N, Lopes RB, Fujiwara K. Tyrosine phosphorylation of platelet endothelial cell adhesion molecule-1 (PECAM-1, CD31) in mechanically stimulated vascular endothelial cells. Eur JCell Biol. 1997;72(3):229-237.

31. Tzima E, et al. A mechanosensory complex that mediates the endothelial cell response to fluid shear stress. Nature. 2005;437(7057):426-431.

32. Conway DE, Breckenridge MT, Hinde E, Gratton E, Chen CS, Schwartz MA. Fluid shear stress on endothelial cells modulates mechanical tension across VE-cadherin and PECAM-1. Curr Biol.
2013;23(11):1024-1030

33. Coon BG, et al. Intramembrane binding of VEcadherin to VEGFR2 and VEGFR3 assembles the endothelial mechanosensory complex. J Cell Biol. 2015;208(7):975-986.

34. Orr AW, Sanders JM, Bevard M, Coleman E, Sarembock IJ, Schwartz MA. The subendothelial extracellular matrix modulates NF- $\mathrm{\kappa B}$ activation by flow: a potential role in atherosclerosis. J Cell Biol. 2005;169(1):191-202.

35. Funk SD, Yurdagul A Jr, Green JM, Jhaveri KA, Schwartz MA, Orr AW. Matrix-specific protein kinase A signaling regulates p21-activated kinase activation by flow in endothelial cells. Circ Res. 2010;106(8):1394-1403.

36. Fleming I, Fisslthaler B, Dixit M, Busse R. Role of PECAM- 1 in the shear-stress-induced activation of Akt the endothelial nitric oxide synthase (eNOS) in endothelial cells. J Cell Sci. 2005;118(pt 18):4103-4111.

37. Otte LA, et al. Rapid changes in shear stress induce dissociation of a $\mathrm{G}$ alpha (q/11)-platelet endothelial cell adhesion molecule-1 complex. JPhysiol. 2009;587(pt 10):2365-2373.

38. Rodbard S. Vascular caliber. Cardiology. 1975;60(1):4-49.

39. Dajnowiec D, Langille BL. Arterial adaptations to chronic changes in haemodynamic function: coupling vasomotor tone to structural remodelling. Clin Sci (Lond). 2007;113(1):15-23.

40. Buschmann I, Heil M, Jost M, Schaper W. Influence of inflammatory cytokines on arteriogenesis. Microcirculation. 2003;10(3-4):371-379.

41. Novak ML, Koh TJ. Phenotypic transitions of macrophages orchestrate tissue repair. Am J Pathol. 2013;183(5):1352-1363.

42. Chen Z, Rubin J, Tzima E. Role of PECAM-1 in arteriogenesis specification of preexisting collaterals. Circ Res. 2010;107(11):1355-1363.

43. Chen Z, Tzima E. PECAM- 1 is necessary for flow-induced vascular remodeling. Arterioscler Thromb Vasc Biol. 2009;29(7):1067-1073.

44. Langille BL. Arterial remodeling: relation to hemodynamics. Can J Physiol Pharmacol. 1996;74(7):834-841.

45. Dolan JM, Sim FJ, Meng H, Kolega J. Endothelial cells express a unique transcriptional profile under very high wall shear stress known to induce expansive arterial remodeling. Am JPhysiol Cell Physiol. 2012;302(8):C1109-C1118.

46. Castier Y, Brandes RP, Leseche G, Tedgui A, Lehoux S. p47phox-dependent NADPH oxidase regulates flow-induced vascular remodeling. Circ Res. 2005;97(6):533-540.

47. Chatzizisis YS, Coskun AU, Jonas M, Edelman ER, Feldman CL, Stone PH. Role of endothelial shear stress in the natural history of coronary atherosclerosis and vascular remodeling: molecular, 
cellular, and vascular behavior. J Am Coll Cardiol. 2007;49(25):2379-2393.

48. Chiu JJ, Usami S, Chien S. Vascular endothelial responses to altered shear stress: pathologic implications for atherosclerosis. Ann Med. 2009;41(1):19-28.

49. Chiu JJ, Chien S. Effects of disturbed flow on vascular endothelium: pathophysiological basis and clinical perspectives. Physiol Rev. 2011;91(1):327-387.

50. Markl M, Brendecke SM, Simon J, Barker AJ, Weiller C, Harloff A. Co-registration of the distribution of wall shear stress and 140 complex plaques of the aorta. Magn Reson Imaging. 2013;31(7):1156-1162.

51. Knight J, et al. Choosing the optimal wall shear parameter for the prediction of plaque location-A patient-specific computational study in human right coronary arteries. Atherosclerosis. 2010;211(2):445-450

52. Peiffer V, Sherwin SJ, Weinberg PD. Computation in the rabbit aorta of a new metric - the transverse wall shear stress - to quantify the multidirectional character of disturbed blood flow. J Biomech. 2013;46(15):2651-2658.

53. Davies PF, Civelek M, Fang Y, Fleming I. The atherosusceptible endothelium: endothelial phenotypes in complex haemodynamic shear stress regions in vivo. Cardiovasc Res. 2013;99(2):315-327.

54. Wang C, Baker BM, Chen CS, Schwartz MA. Endothelial cell sensing of flow direction. Arterioscler Thromb Vasc Biol. 2013;33(9):2130-2136.

55. Mohan S, Mohan N, Sprague EA. Differential activation of NF- $\mathrm{NB}$ in human aortic endothelial cells conditioned to specific flow environments. Am J Physiol. 1997;273(2 pt 1):C572-C578.

56. Liu Y, et al. A novel pathway spatiotemporally activates Rac1 and redox signaling in response to fluid shear stress. JCell Biol. 2013;201(6):863-873.

57. Baeyens N, et al. Syndecan 4 is required for endothelial alignment in flow atheroprotective signaling. Proc Natl Acad Sci U S A. 2014;111(48):17308-17313.

58. Alexy T, James AM1, Searles CD2. Shear sensitive microRNAs and atherosclerosis. Biorheology. 2014;51(2-3):147-158.

59. Hosin AA, Prasad A, Viiri LE, Davies AH, Shalhoub J. MicroRNAs in atherosclerosis. J Vasc Res. 2014;51(5):338-349.

60. Xiao H, et al. Sterol regulatory element binding protein 2 activation of NLRP3 inflammasome in endothelium mediates hemodynamic-induced atherosclerosis susceptibility. Circulation. 2013;128(6):632-642.

61. Ridker PM, et al. Effects of interleukin-1 $\beta$ inhibition with canakinumab on hemoglobin A1c, lipids, C-reactive protein, interleukin-6, fibrinogen: a phase IIb randomized, placebo-controlled trial. Circulation. 2012;126(23):2739-2748.

62. Chen Z, et al. Oxidative stress activates endothelial innate immunity via sterol regulatory element binding protein 2 (SREBP2) transactivation of microRNA-92a. Circulation. 2015;131(9):805-814.

63. Chiang HY, Korshunov VA, Serour A, Shi F, Sottile J. Fibronectin is an important regulator of flow-induced vascular remodeling. Arterioscler Thromb Vasc Biol. 2009;29(7):1074-1079.

64. Kim S, Bell K, Mousa SA, Varner JA. Regulation of angiogenesis in vivo by ligation of integrin $\alpha 5 \beta 1$ with the central cell-binding domain of fibronectin.
Am J Pathol. 2000;156(4):1345-1362.

65. Astrof S, Hynes RO. Fibronectins in vascular morphogenesis. Angiogenesis. 2009;12(2):165-175.

66. Orr AW, Sanders JM, Bevard M, Coleman E, Sarembock IJ, Schwartz MA. The subendothelial extracellular matrix modulates NF- $\kappa$ B activation by flow: a potential role in atherosclerosis. J Cell Biol. 2005;169(1):191-202.

67. Hahn C, Orr AW, Sanders JM, Jhaveri KA, Schwartz MA. The subendothelial extracellular matrix modulates JNK activation by flow. Circ Res. 2009;104(8):995-1003.

68. Yurdagul A Jr, Green J, Albert P, McInnis MC, Mazar AP, Orr AW. $\alpha 5 \beta 1$ integrin signaling mediates oxidized low-density lipoprotein-induced inflammation early atherosclerosis. Arterioscler Thromb Vasc Biol. 2014;34(7):1362-1373.

69. Yurdagul A Jr, Chen J, Funk SD, Albert P, Kevil CG, Orr AW. Altered nitric oxide production mediates matrix-specific PAK2 and NF- $\kappa \mathrm{B}$ activation by flow. Mol Biol Cell. 2013;24(3):398-408.

70. Tan MH, Sun Z, Opitz SL, Schmidt TE, Peters JH, George EL. Deletion of the alternatively spliced fibronectin EIIIA domain in mice reduces atherosclerosis. Blood. 2004;104(1):11-18.

71. Babaev VR, Porro F, Linton MF, Fazio S, Baralle FE, Muro AF. Absence of regulated splicing of fibronectin EDA exon reduces atherosclerosis in mice. Atherosclerosis. 2008;197(2):534-540.

72. Rohwedder I, et al. Plasma fibronectin deficiency impedes atherosclerosis progression and fibrous cap formation. EMBO Mol Med. 2012;4(7):564-576.

73. Green J, Yurdagul A Jr, McInnis MC, Albert P, Orr AW. Flow patterns regulate hyperglycemia-induced subendothelial matrix remodeling during early atherogenesis. Atherosclerosis. 2014;232(2):277-284.

74. Smedby O. Do plaques grow upstream or downstream? An angiographic study in the femoral artery. Arterioscler Thromb Vasc Biol. 1997;17(5):912-918.

75. Gijsen F, van der Giessen A, van der Steen A, Wentzel J. Shear stress and advanced atherosclerosis in human coronary arteries. J Biomech. 2013;46(2):240-247.

76. Cheng C, et al. Atherosclerotic lesion size and vulnerability are determined by patterns of fluid shear stress. Circulation. 2006;113(23):2744-2753.

77. Phinikaridou A, Hua N, Pham T, Hamilton JA. Regions of low endothelial shear stress colocalize with positive vascular remodeling and atherosclerotic plaque disruption: an in vivo magnetic resonance imaging study. Circ Cardiovasc Imaging. 2013;6(2):302-310.

78. Chatzizisis YS, et al. Augmented expression and activity of extracellular matrix-degrading enzymes in regions of low endothelial shear stress colocalize with coronary atheromata with thin fibrous caps in pigs. Circulation. 2011;123(6):621-630.

79. Koskinas KC, et al. Thin-capped atheromata with reduced collagen content in pigs develop in coronary arterial regions exposed to persistently low endothelial shear stress. Arterioscler Thromb Vasc Biol. 2013;33(7):1494-1504.

80. Pedrigi RM, de Silva R, Bovens SM, Mehta VV, Petretto E, Krams R. Thin-cap fibroatheroma rupture is associated with a fine interplay of shear and wall stress. Arterioscler Thromb Vasc Biol. 2014;34(10):2224-2231.

81. Kelly-Arnold A, Maldonado N, Laudier D, Aikawa E, Cardoso L, Weinbaum S. Revised microcalcification hypothesis for fibrous cap rupture in human coronary arteries. Proc Natl Acad Sci U S A. 2013;110(26):10741-10746.

82. Glagov S, Weisenberg E, Zarins CK, Stankunavicius R, Kolettis GJ. Compensatory enlargement of human atherosclerotic coronary arteries. N Engl JMed. 1987;316(22):1371-1375.

83. Korshunov VA, Schwartz SM, Berk BC. Vascular remodeling: hemodynamic and biochemical mechanisms underlying Glagov's phenomenon. Arterioscler Thromb Vasc Biol. 2007;27(8):1722-1728.

84. Sommer G, Regitnig P, Költringer L, Holzapfel GA. Biaxial mechanical properties of intact and layerdissected human carotid arteries at physiological and supraphysiological loadings. Am J Physiol Heart Circ Physiol. 2010;298(3):H898-H912.

85. Mulligan-Kehoe MJ, Simons M. Vasa vasorum in normal and diseased arteries. Circulation. 2014;129(24):2557-2566.

86. Chen PY, et al. Endothelial-to-mesenchymal transition drives atherosclerosis progression. J Clin Invest. 2015;125(12):4514-4528.

87. Moonen JR, et al. Endothelial-to-mesenchymal transition contributes to fibro-proliferative vascular disease and is modulated by fluid shear stress. Cardiovasc Res. 2015;108(3):377-386.

88. Cooley BC, et al. TGF- $\beta$ signaling mediates endothelial-to-mesenchymal transition (EndMT) during vein graft remodeling. Sci Transl Med. 2014;6(227):227ra34

89. Varnava AM, Mills PG, Davies MJ. Relationship between coronary artery remodeling and plaque vulnerability. Circulation. 2002;105(8):939-943.

90. Maehara A, et al. Morphologic and angiographic features of coronary plaque rupture detected by intravascular ultrasound. J Am Coll Cardiol. 2002;40(5):904-910.

91. Inaba S, et al. Impact of positive and negative lesion site remodeling on clinical outcomes: insights from PROSPECT. JACC CardiovasC Imaging. 2014;7(1):70-78.

92. Seiler C, Stoller M, Pitt B, Meier P. The human coronary collateral circulation: development and clinical importance. Eur Heart J. 2013;34(34):2674-2682.

93. Faber JE, Chilian WM, Deindl E, van Royen N, Simons M. A brief etymology of the collateral circulation. Arterioscler Thromb Vasc Biol. 2014;34(9):1854-1859.

94. Mac Gabhann F, Peirce SM. Collateral capillary arterialization following arteriolar ligation in murine skeletal muscle. Microcirculation. 2010;17(5):333-347.

95. Orr AW, Sanders JM, Bevard M, Coleman E, Sarembock IJ, Schwartz MA. The subendothelial extracellular matrix modulates NF-kappa B activation by flow: a potential role in atherosclerosis. JCell Biol. 2005;169(1):191-202.

96. Morrison AR, et al. Chemokine-coupled $\beta 2$ integrin-induced macrophage Rac2-Myosin IIA interaction regulates VEGF-A mRNA stability arteriogenesis. J Exp Med. 2014;211(10):1957-1968.

97. Fung E, Helisch A. Macrophages in collateral arteriogenesis. Front Physiol. 2012;3:353. 
98. Uhlemann M, et al. Impact of different exercise training modalities on the coronary collateral circulation and plaque composition in patients with significant coronary artery disease (EXCITE trial): study protocol for a randomized controlled trial. Trials. 2012;13:167.

99. Buschmann EE, et al. Improvement of fractional flow reserve and collateral flow by treatment with external counterpulsation (Art.Net.-2 Trial). Eur JClin Invest. 2009;39(10):866-875.

100. Gloekler S, et al. Coronary collateral growth by external counterpulsation: a randomised controlled trial. Heart. 2010;96(3):202-207.

101. Chalothorn D, Faber JE. Strain-dependent variation in collateral circulatory function in mouse hindlimb. Physiol Genomics. 2010;42(3):469-479.

102.Sealock R, Zhang H, Lucitti JL, Moore SM, Faber JE. Congenic fine-mapping identifies a major causal locus for variation in the native collateral circulation and ischemic injury in brain and lower extremity. Circ Res. 2014;114(4):660-671.

103. Tirziu D, et al. Delayed arteriogenesis in hypercholesterolemic mice. Circulation. 2005;112(16):2501-2509.

104.van Weel V, et al. Hypercholesterolemia reduces collateral artery growth more dominantly than hyperglycemia or insulin resistance in mice. Arterioscler Thromb Vasc Biol. 2006;26(6):1383-1390.
105. DiStasi MR, et al. Impaired compensation to femoral artery ligation in diet-induced obese mice is primarily mediated via suppression of collateral growth by Nox2 and p47phox. Am J Physiol Heart Circ Physiol. 2015;309(7):H1207-H1217.

106. Faber JE, et al. Aging causes collateral rarefaction and increased severity of ischemic injury in multiple tissues. Arterioscler Thromb Vasc Biol. 2011;31(8):1748-1756

107. Clatterbuck RE, Eberhart CG, Crain BJ, Rigamonti D. Ultrastructural and immunocytochemical evidence that an incompetent blood-brain barrier is related to the pathophysiology of cavernous malformations. J Neurol Neurosurg Psychiatry. 2001;71(2):188-192.

108. Draheim KM, Fisher OS, Boggon TJ, Calderwood DA. Cerebral cavernous malformation proteins at a glance. JCell Sci. 2014;127(pt 4):701-707.

109. Friedlander RM. Clinical practice. Arteriovenous malformations of the brain. NEngl J Med. 2007;356(26):2704-2712.

110.Zawistowski JS, et al. CCM1 and CCM2 protein interactions in cell signaling: implications for cerebral cavernous malformations pathogenesis. Hum Mol Genet. 2005;14(17):2521-2531.

111. Kleaveland B, et al. Regulation of cardiovascular development and integrity by the heart of glass-cerebral cavernous malformation protein pathway. Nat Med.2009;15(2):169-176.

112. Fischer A, Zalvide J, Faurobert E, Albiges-Rizo C, Tournier-Lasserve E. Cerebral cavernous malformations: from CCM genes to endothelial cell homeostasis. Trends Mol Med. 2013;19(5):302-308.

113. Zhou Z, et al. The cerebral cavernous malformation pathway controls cardiac development via regulation of endocardial MEKK3 signaling KLF expression. Dev Cell. 2015;32(2):168-180.

114. Atkins GB, Jain MK. Role of Kruppel-like transcription factors in endothelial biology. Circ Res. 2007;100(12):1686-1695.

115. Maddaluno L, et al. EndMT contributes to the onset and progression of cerebral cavernous malformations. Nature. 2013;498(7455):492-496.

116. Faurobert E, et al. CCM1-ICAP-1 complex controls beta1 integrin-dependent endothelial contractility fibronectin remodeling. J Cell Biol. 2013;202(3):545-561.

117. Ross TD, et al. Integrins in mechanotransduction. Curr Opin Cell Biol. 2013;25(5):613-618.

118. Macek Jilkova Z, et al. CCM proteins control endothelial beta1 integrin dependent response to shear stress. Biol Open. 2014;3(12):1228-1235.

119. Renz M, et al. Regulation of $\beta 1$ integrin-Klf2mediated angiogenesis by CCM proteins. Dev Cell. 2015;32(2):181-190. 